\title{
Compressive-shear adhesion characterization of polyvinyl-butyral and ethylene-vinyl acetate at different curing times before and after exposure to damp-heat conditions
}

\author{
Valentin Chapuis* , Ségolène Pélisset, Marylène Raeis-Barnéoud, Heng-Yu Li, \\ Christophe Ballif and Laure-Emmanuelle Perret-Aebi \\ Photovoltaics and Thin Film Electronics Laboratory, Ecole Polytechnique Fédérale de Lausanne (EPFL), Rue A.-L. Breguet 2, CH-2000 \\ Neuchâtel, Switzerland
}

\begin{abstract}
Photovoltaic (PV) module efficiency and reliability are two factors that have an important impact on the final cost of the PV electricity production. It is widely accepted that a good adhesion between the encapsulant and the different substrates of a PV module is needed to ensure long-term reliability. Several testing procedures exist that use a metric derived from the force at interface failure to characterize the adhesion. It has, however, not been demonstrated that those metrics relate directly to the interfacial adhesion (defined as the surface energy density needed to break interfacial bonds), and the obtained results usually relate to an apparent adhesion strength. In this work, we describe a new design for compressive-shear testing of polymer layers bonded to rigid substrates. We use it to characterize real interfacial adhesion of ethylene-vinyl acetate (EVA) and polyvinyl-butyral (PVB) to a glass substrate before and after degradation in damp-heat. Our results show that a peak-force based metric is unable to capture the evolution of adhesion through degradation, and a new metric based on the elastic strain energy of the encapsulant is proposed. Moreover, we show that PVB adhesion to glass is much more affected by damp-heat exposure where polymer saturation takes place, in comparison with the adhesion of EVA to glass. The presented characterization protocol is a powerful tool that can help in assessing the reliability of an encapsulant facing specific degradation conditions. Copyright (C) 2012 John Wiley \& Sons, Ltd.
\end{abstract}

KEYWORDS

photovoltaic; reliability; adhesion; polymer; compressive shear

* Correspondence

Valentin Chapuis, Photovoltaics and Thin Film Electronics Laboratory, Ecole Polytechnique Fédérale de Lausanne (EPFL), Rue A.-L. Breguet 2, CH-2000 Neuchâtel, Switzerland.

Email: valentin.chapuis@epfl.ch

Received 23 February 2012; Revised 16 May 2012; Accepted 26 June 2012

\section{INTRODUCTION}

Increasing module lifetime (typically over 25 years) is a key toward photovoltaic (PV) electricity cost reduction. Therefore PV devices must be protected against environmental stresses (e.g., ultra-violet radiation, rain, moisture, hail, thermal cycling) [1]. This protection is achieved through a packaging step in which the PV device is encapsulated between glasses and/or foils by the mean of one or two polymer layers. It is well-known that the quality and durability of this protective system is dependent on the adhesion between the different layers [2,3]. A degradation of the adhesion between the different layers of the encapsulation system may, for example, lead to accelerated moisture ingress resulting in reduced electrical performances of the device or to a delamination failure [4,5]. Additionally, poor adhesion may prevent PV modules from successfully passing certain IEC standards such as the breakage test described in the IEC 61730 [6]. Therefore, adhesion characterization and optimization is of primary interest in the PV field. Over the years, several testing procedures to characterize adhesion in different systems have been developed in various application fields such as composite testing [7,8], fracture mechanics [9], polymer science [10], aerospace [11] and more recently in the PV field to evaluate the adhesion of a polymeric layer to rigid or flexible substrates. The most common ones such as 
peel tests [12,13], lap shear tests [14-16] or compressive shear tests (CST) $[17,18]$ are extensively described in the literature.

The mentioned testing procedures usually consist of recording the force acting on the sample as a function of the applied displacement, resulting in joint response curves as shown in Figure 1. From those curves, several indicators can be extracted that are intended to describe the interfacial adhesion between the polymeric layer and the substrates. Most common ones are the mean force at which delamination stably propagates divided by the sample width $[\mathrm{N} / \mathrm{m}]$ for peel tests and the peak force at joint failure normalized by the sample surface $\left[\mathrm{N} / \mathrm{mm}^{2}\right]$ for lap shear and compressive shear tests.

However, no direct correlation has been made between the usual indicators and the "interfacial adhesion" defined as the energy density required to break interfacial bonds. The usual indicators relate to an apparent joint strength and might be influenced by several parameters including sample geometry $[19,20]$ or adhesive and substrate bulk properties $[21,22]$.

The present paper first describes a particular CST design. The different adhesion indicators that can be derived from this testing procedure are then presented and a short summary of the bonding chemistry of ethylene vinyl acetate (EVA) and polyvinyl-butyral (PVB) on glass is given to help the reader in understanding the subsequent discussion. The CST is then used to characterize the adhesion of PVB and EVA on glass before and after degradation in damp-heat (DH) conditions $\left(85^{\circ} \mathrm{C} / 85 \% \mathrm{RH}\right)$. The influence of the EVA curing time on adhesion and its evolution after degradation is investigated. Finally, a new metric based on the stored elastic energy is proposed for an accurate description of the adhesion.

\section{MATERIALS AND METHODS}

\subsection{Compressive shear test}

Standard CST involves three main components (Figure 2). An upper fixture on which a compressive loading is applied (often through a sphere-on-flat arrangement that ensures transmission of vertical forces only), a lower fixture that can translate perpendicular to the loading

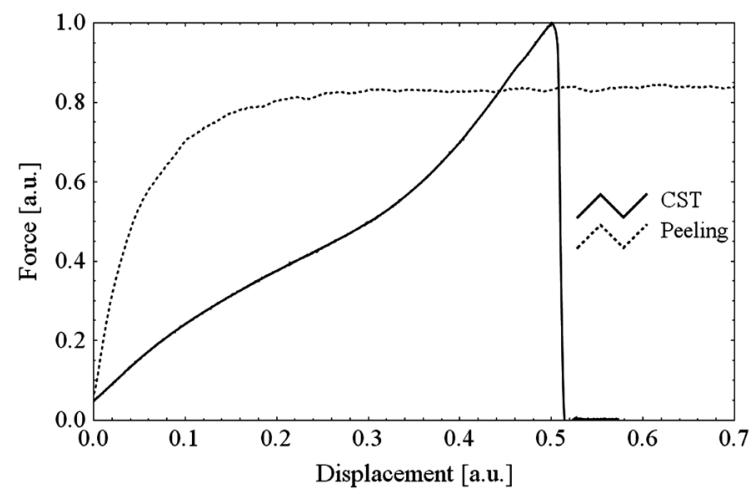

Figure 1. Typical joint response curves for peel test (dashed) and compressive shear test (plain), showing the evolution of the force acting on the joint as a function of the applied displacement.
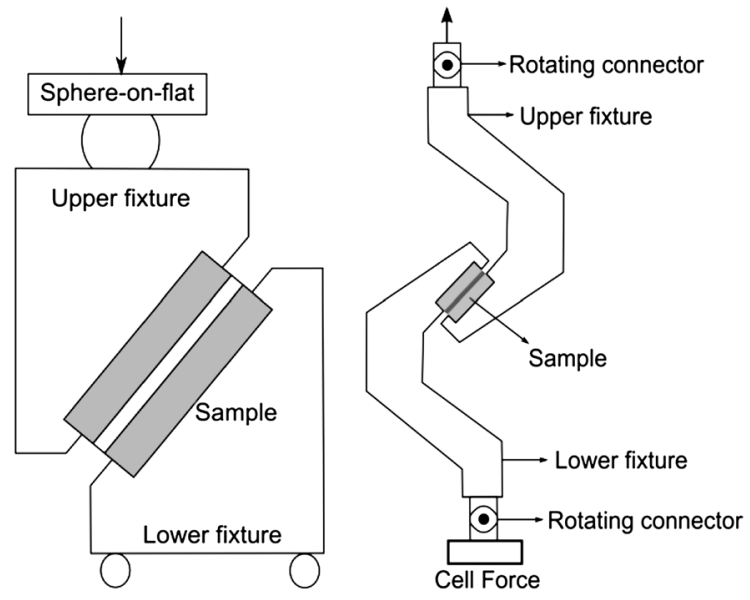

Figure 2. (Left) Standard compressive shear test (CST), and (right) adaptation of CST setup for a testing machine working in tension.

direction and a plane-parallel sample consisting of an adhesive layer (encapsulant) laminated in between two rigid substrates (glass). The sample is oriented at a given angle with respect to the loading direction so that it experiences a combination of both shear and compressive stresses. The compressive contribution ensures that neither sample misalignment nor peel stress develops during the test, resulting in improved reproducibility (Figure 3 ) as compared with other testing procedures (peel or lap-shear). Force and applied displacement are recorded until failure. At a loading angle of $45^{\circ}$, the compressive stress equals the shear stress. Considering the adhesive layer as nearly incompressible, the compressive stress results in a negligible strain. The sample can therefore be assumed to undergo pure shear strain.

Our new CST system (Figure 2) consists of a modified fixture geometry that allows using a testing machine working in tension and not in compression. The sample is fixed in-between two loading arms connected to the cell force, the machine moving arm respectively, through two rotating connectors. The rotating connectors allow self-alignment of the loading arms, and the sample throughout the test ensures that no direct peel stress is developed. Finite element analyses (FEA) using the Abaqus 6.8-1 commercial code [23] were carried out confirming that this particular fixture geometry results in the same stress distribution on the adhesive/adherend interface as the standard setup (Figure 3). In the FEA, both polymer (p) and glass (g) were modeled as isotropic linear elastic materials with Young's modulus of $\mathrm{E}_{\mathrm{p}}=100 \mathrm{MPa}$ and $\mathrm{E}_{\mathrm{g}}=70 \mathrm{GPa}$, and Poisson coefficients of $v_{p}=0.45$ and $v_{g}=0.22$, respectively. The model represents a two-dimensional (2D) section of the CST sample modeled in plane strain using a glass thickness of $3 \mathrm{~mm}$ and an adhesive layer thickness of $0.5 \mathrm{~mm}$ for a sample length of $25 \mathrm{~mm}$. The mesh consists of 18500 eight-node biquadratic plane strain quadrilateral, reduced integration CPE8R finite elements. In this way, 20 elements are placed in the thickness of the adhesive layer.

In our new design, the sample is loaded through a displacement controlled procedure at a given arm 

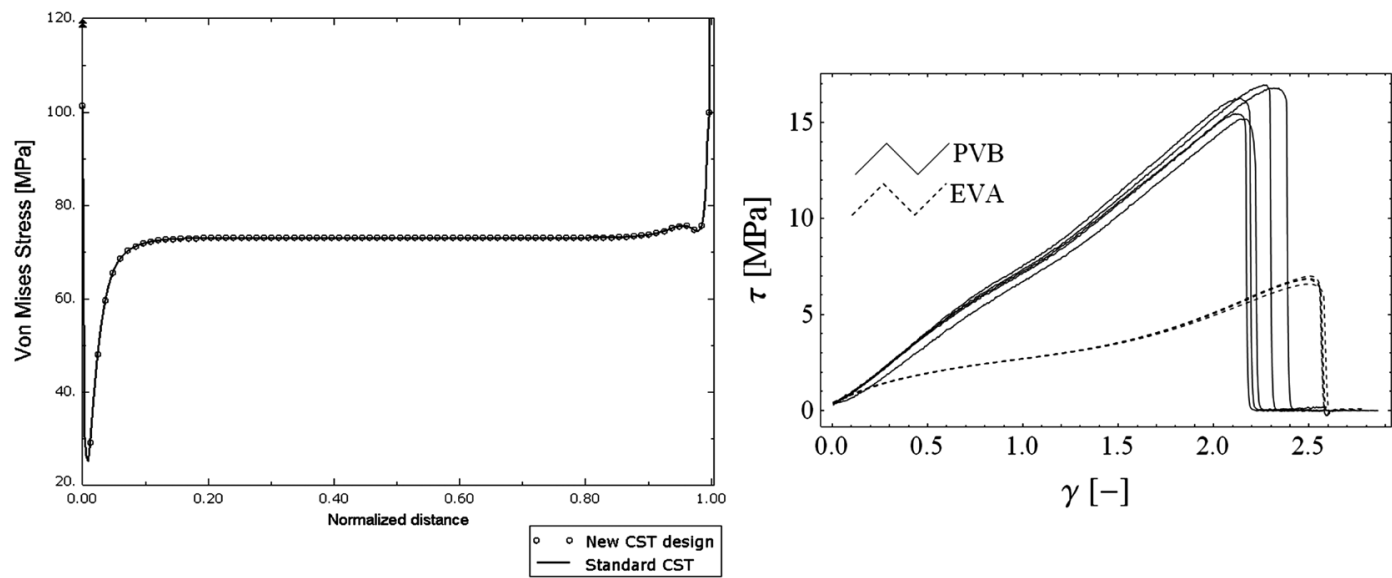

Figure 3. (Left) Von Mises stress profiles along the upper substrate/polymer interface on Figure 2, in a standard compressive shear test (CST) design (plain curve) and in our new design (dots) as obtained from FEA. (Right) Typical reproducibility of our CST for polyvinyl-butyral and ethylene-vinyl acetate bonded to glass.

displacement velocity on a Schenck-Trebel RSA-100 tensile testing machine (Schenck-Trebel Corp., Farmingdale, NY, USA), combined with a 10 or $100 \mathrm{kN}$ cell force. A $100 \mathrm{~Hz}$ acquisition frequency was used, and the resulting data was smoothed using a moving average function to get rid of the cell force acquisition noise. The arm displacement velocity is calculated based on the adhesive layer thickness in order to induce a constant shear strain rate for all samples. Equation (1) gives the relationship between imposed vertical displacement $u_{\mathrm{y}}$ and global shear strain $\gamma$, under the assumption of small deformations (Figure 4). The arm displacement velocity $v_{\mathrm{y}}$ is then obtained as a function of the shear rate $\dot{\gamma}$ using a time derivative of this relationship (2). A shear rate of $0.2 \mathrm{~s}^{-1}$ is chosen as a standard solicitation rate. The acquisition of force and displacement values is achieved using a data acquisition module USB-6009 from National Instruments combined with a homemade LabView procedure.

$$
\begin{gathered}
u_{y}=\frac{1}{2} \frac{\gamma \cdot h_{0}}{\cos (\theta)} \\
v_{y}=\frac{\partial u_{y}}{\partial t}=\frac{1}{2} \frac{\dot{\gamma} \cdot h_{0}}{\cos (\theta)}
\end{gathered}
$$

The tests are performed on square samples $\left(25 \times 25 \mathrm{~mm}^{2}\right)$ consisting of a polymeric layer laminated in-between two pieces of $2.9 \mathrm{~mm}$ thick glass in a vacuum laminator. The thickness of the adhesive layer is in the range of $400-800 \mu \mathrm{m}$, and the glass is washed with isopropanol and rinsed with de-ionized water before lamination. The samples are first processed in a $100 \times 100 \mathrm{~mm}^{2}$ format and then cut into $25 \times 25 \mathrm{~mm}^{2}$ pieces by means of a diamond sawing process. The purpose of this required preparation technique is to provide samples with four equivalent defect-free edges and a homogeneous adhesive thickness, ensuring high measurement reproducibility. The CSTs presented in this paper were performed at room temperature.

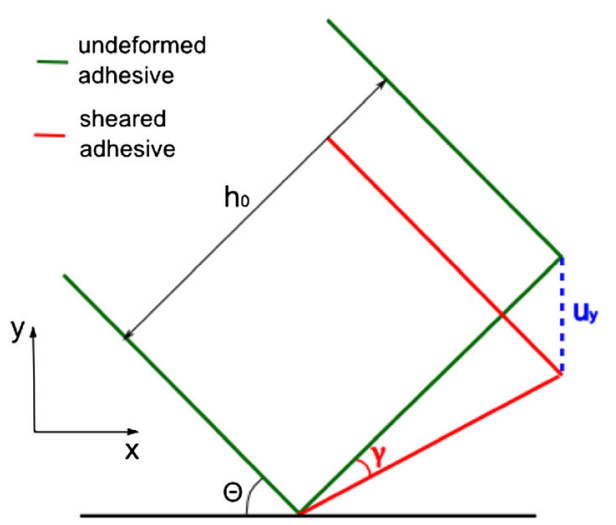

Figure 4. Sketch of the adhesive deformation used to relate the vertical displacement $u_{y}$ to the shear strain $\gamma$ for an adhesive layer of thickness $h_{0}$ loaded at an angle $\theta$.

\section{THEORY AND CALCULATIONS}

\subsection{Adhesion indicators}

When a CST is performed, the adhesive layer deforms in shear until failure of one interface. The delamination can be seen to be either force-controlled (i.e., a critical force has to be exceeded to induce delamination), displacementcontrolled or energy-controlled. Depending on the failure criterion that is used to describe the delamination, different metrics are calculated from the acquired force-displacement curves in order to characterize adhesion.

\subsubsection{Force controlled indicator.}

The peak force at which sudden delamination occurs is normalized by the sample surface (lap-shear) or the sample width (peeling) to describe the force density needed to induce failure. This indicator, often referred to as "adhesion strength" for lap-shear tests or "peeling strength" for peeling 
tests, is widely used in the PV community to describe adhesion of encapsulants to rigid substrates [18,24].

A more accurate force-controlled indicator can be obtained considering only the shearing component $F_{\text {shear }}$ of the force $F$ acting on the sample. This contribution depends on the loading angle $\theta\left(45^{\circ}\right.$ in the present case) and can be calculated as in (3)

$$
F_{\text {shear }}=F \cdot \cos (\theta) \stackrel{\theta=45^{\circ}}{=} F \cdot \frac{\sqrt{2}}{2}
$$

Assuming that the shearing component results in a homogeneous shear deformation, an "equivalent peak shear stress" $\left(\tau_{\max }\right)$ can be calculated using Equation (4), where $S_{\mathrm{del}}$ is the delaminated area.

$$
\tau_{\max }=\frac{F_{\text {shear }}}{S_{\text {del }}}
$$

The advantages of this metric as compared with the standard peak force density are twofold. First, taking into account only the shearing component allows a comparison between CST performed at different angles $\theta$. Second, the normalization surface is the real delaminated area allowing a more accurate description especially when partial delamination occurs. This metric will be referred to as "peak stress" in the following sections and is expressed in [MPa].

\subsubsection{Displacement controlled indicator.}

The maximum equivalent shear strain $\gamma_{\max }$ corresponds to a homogeneous pure shear strain calculated at the peak stress level. It is calculated from the displacement at peak force $d_{F \max }$ using (5). This metric takes into account the thickness of the encapsulant allowing a comparison between various samples with varying encapsulant thicknesses.

$$
\gamma_{\max }=\frac{2 \cdot d_{F_{\max }} \cos (\theta)}{h_{0}}
$$

This metric aims to represent the maximum shear deformation that the joint can sustain before delamination. It is expressed in radians and will be referred hereafter as "peak strain".

\subsubsection{Energy controlled indicator.}

Transforming a CST curve from force-displacement to a shear stress-shear strain relationship using (3), (4) and (5) provides results independent of both the encapsulant thickness and the loading angle.

Moreover, the encapsulant layer is much more compliant than the substrates, the elastic modulus of EVA being three orders of magnitude lower than that of glass. It can thus be assumed that the imposed displacement is only accommodated by the shearing deformation of the encapsulant. Consequently, integrating this stress-strain curve (6) leads to a deformation energy density $E^{\mathrm{el}}$ expressed in $\left[\mathrm{mJ} / \mathrm{mm}^{3}\right]$, which takes into account both the elastic and plastic deformation regimes of the encapsulant. If no plastic dissipation occurs, the deformation energy density represents the energy available for delamination.

$$
E^{\mathrm{el}}=\int \tau(\gamma) \mathrm{d} \gamma
$$

Using the energy-based failure criterion, the sample delamination is seen to occur when a critical value of elastic energy density is stored in the polymer layer. This metric will be called "stored energy" in the next sections and is expressed in $\left[\mathrm{mJ} / \mathrm{mm}^{3}\right]$.

\subsection{Ethylene-vinyl acetate and polyvinyl- butyral adhesion to glass}

We present here a short synthesis of the mechanisms of adhesion for EVA and PVB. It contains elements required for the reader to better understand the following discussion.

The adhesion of an EVA layer to a glass substrate [25] is performed by using adhesion promoters called silanes that are present in the bulk of the polymer. Commercially available silanes are usually alkoxysilanes in which alkoxy reactive groups bond to the EVA chains (grafting reaction) whereas the silanol groups bond to the glass substrate.

\subsubsection{Reaction of silane with glass.}

The typical reaction of silane (typically 3-(trimethoxysilyl) propyl ester methacrylic acid in the case of EVA) with glass occurs in three steps (Figure 5). The alkoxysilane is first hydrolyzed to generate a silanol intermediate that can partially oligomerize in solution. In the second step, hydrogen bonding occurs, followed by elimination of water (condensation reaction) generating a stable $\mathrm{Si}-\mathrm{O}-\mathrm{Si}$ bond. The bonds established between silane and glass are thus mostly covalent in nature and form what will be called hereafter the interface between EVA and glass.

\subsubsection{Grafting reaction of the adhesion promoters to the ethylene-vinyl acetate chain.}

Grafting of the silane coupling agent onto the EVA chains is performed through a radical reaction induced by thermally activated peroxides. These peroxides (typically tert-butylperoxy-2-ethylhexyl carbonate) are added to the EVA formulation to initiate the crosslinking reaction. Therefore, the curing process of EVA and the grafting reaction occurs in parallel during the lamination process. Figure 6 represents the typical situation of the radical reaction involved during EVA processing [26].

The grafting of silane onto EVA thus forms a new layer, which will be called "interphase" in what follows. The adhesion of EVA depends then on both the interface (silane/glass) and interphase (silane/EVA) quality.

It must be noticed that the peroxide concentration in the bulk EVA is higher than that needed to complete the curing reaction. Moreover, peroxide activation energy is around $120 \mathrm{~kJ} / \mathrm{mol}$ [26] corresponding to an optimal activation temperature of $448 \mathrm{~K}\left(175^{\circ} \mathrm{C}\right)$. Therefore, as standard EVA lamination takes place at $\sim 140{ }^{\circ} \mathrm{C}$, a non-negligible amount of peroxide remains in the encapsulant after lamination, even for high curing times [27,28]. 
Hydrolysis

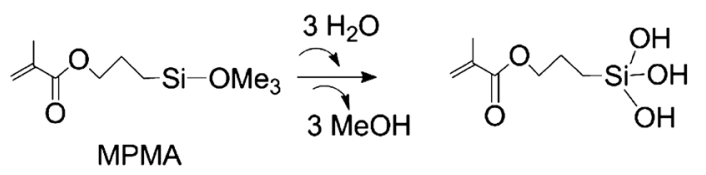

Hydrogen bonding

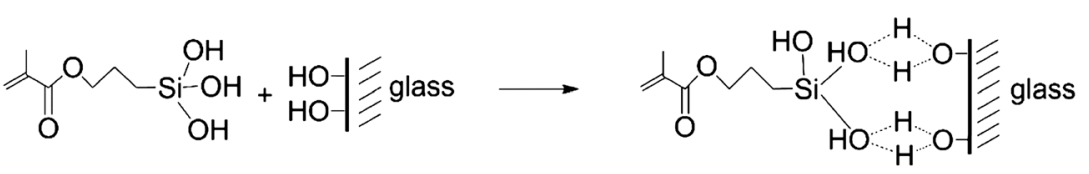

Condensation

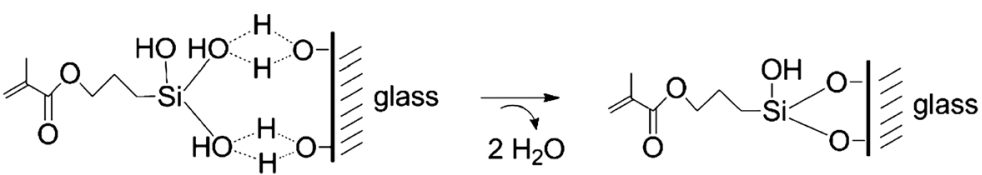

Figure 5. Description of the main reactions involved in covalent bonding of silane (typically 3-(trimethoxysilyl)propyl ester methacrylic acid) adhesion promoter to the glass surface.

\subsubsection{Polyvinyl-butyral adhesion to glass.}

Bonding of PVB to glass is somewhat simpler. As PVB is a copolymer of poly vinyl butyral (80\%wt) and poly vinyl alcohol (20\%wt) groups (Figure 7), its bonding to glass does not require any additional adhesion promoters. The polar alcohol group can directly form hydrogen bonds with the glass substrate. As compared with EVA, condensation of the hydrogen-bonded network to form covalent bonds is unlikely. Consequently, adhesion of PVB on glass is mostly related to dispersive forces, even if hydrogen bonds condensation can occur to some extent.

\section{RESULTS}

\subsection{Effect of ethylene-vinyl acetate curing time on adhesion before and after degradation}

Compressive shear test samples containing a layer of EVA and two low-iron glass layers were processed using two lamination cycles involving either a curing time of $300 \mathrm{~s}$ or $900 \mathrm{~s}$ at $140{ }^{\circ} \mathrm{C}(300 \mathrm{~s}$ pre-heating, 300/900 s under one bar, cooling under atmospheric conditions). Glass was always laminated on the tin-free side. For each curing

Initiation step

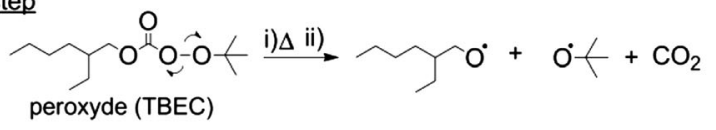

Propagation step
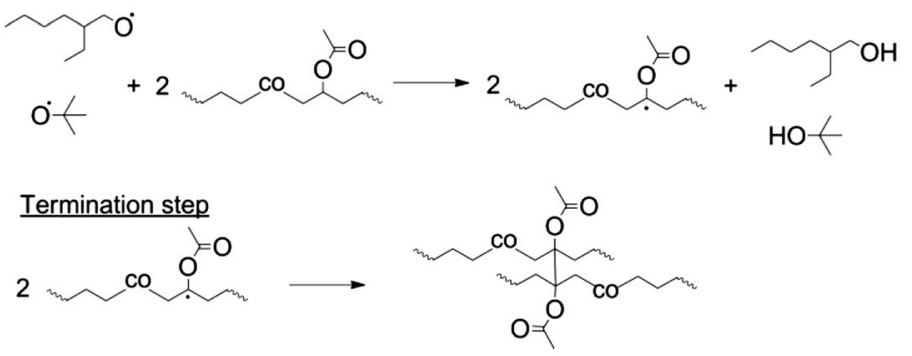

Grafting reaction of the silane on the EVA chain

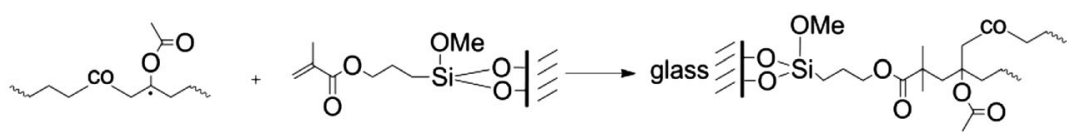

Figure 6. Description of the main steps of the peroxide (typically tert-butylperoxy-2-ethylhexyl carbonate) radical reaction (initiation, propagation and termination) and description of the grafting reaction of silane adhesion promoter onto an ethylene-vinyl acetate copolymer (co) chain. 
(a)

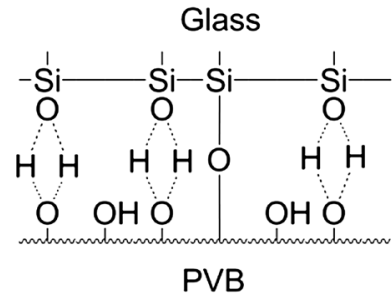

(b)

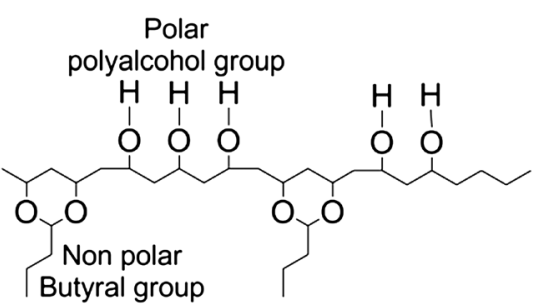

Figure 7. (a) Schematic representation of the interface between polyvinyl-butyral (PVB) and glass, (b) a typical PVB chain with its polar and non-polar groups.

condition, $10-25 \times 25 \mathrm{~mm}^{2}$ samples were produced. Half of the samples were placed in a climate chamber under dampheat conditions $\left(85^{\circ} \mathrm{C}, 85 \% \mathrm{RH}\right)$. The duration of degradation was set to $70 \mathrm{~h}$, which corresponds to saturation of the EVA layer for $30 \mathrm{~h}$. CSTs were then performed on all samples according to the protocol described in Section 2.

Figure 8 shows a set of representative CST response curves for each condition, and Table I summarizes the adhesion indicators and the failure modes.

The curing time of $900 \mathrm{~s}$ corresponds to a standard lamination process during which the degree of crosslinking is maximized. An EVA gel content of over $90 \%$ was measured by using DSC [29]. This value corresponds to a plateau where no significant further curing takes place. The silanes are completely hydrolyzed, and the entire hydrogen network formed by the silanol groups already condensed into covalent bonds. When exposing these samples to DH conditions, one observes an increase of both the peak strain $(+65 \%)$ and the elastic energy $(+70 \%)$, whereas the peak shear stress remains at a constant level around $7 \mathrm{MPa}$. Moreover, the curve shape is modified showing a softer response (lower level of stress needed to induce the same level of strain) in the case of aged samples.

The curing time of $300 \mathrm{~s}$ corresponds to a lower gel content of $65 \%$, which indicates that the crosslinking of EVA is not completed. The bulk EVA is softer, and the failure was cohesive. Thus no evaluation of the adhesion of the $300 \mathrm{~s}$ condition before aging can be made even if the indicators could be calculated. However, after subjecting the 300 s samples to DH, the failure mode changes from cohesive to adhesive, and the CST curve shape is modified, matching the $900 \mathrm{~s}$ behavior before degradation.

\subsection{Evolution of adhesion through damp- heat for polyvinyl-butyral and ethylene- vinyl acetate}

Compressive shear test samples containing a layer of PVB between two low-iron glass layers were processed following the same procedure as that for EVA samples (Section 4.1). The lamination process was different, taking place at $160{ }^{\circ} \mathrm{C}$ in the case of PVB $(10 \mathrm{~min}$ pre-heating, $11 \mathrm{~min}$ under $800 \mathrm{~m}$ bars). Again, half of the samples were placed in a climate chamber under DH conditions for $70 \mathrm{~h}$, undergoing thus the same exposure to $\mathrm{DH}$ conditions as the EVA samples.

Figure 9 shows a set of representative CST response curves for PVB and EVA ( $900 \mathrm{~s}$ of curing). Table II summarizes the adhesion indicators. All samples failed adhesively, and no plasticization of the polymeric layers was observed.

Before degradation, PVB exhibits a higher CST response for all indicators as compared with EVA. However, the values of the indicators drop significantly after $\mathrm{DH}$. The peak stress initially at $16.2 \mathrm{MPa}$ is decreased by a factor of 2, the peak strain drops from 2.2 to 1 , and the stored energy initially at $18.7 \mathrm{~mJ} / \mathrm{mm}^{3}$ is decreased by almost $80 \%$. As mentioned, the situation is different for EVA. The initial response is lower compared with $\mathrm{PVB}$, with a peak stress of $6.8 \mathrm{MPa}$ and a stored energy of $5.5 \mathrm{~mJ} / \mathrm{mm}^{3}$. Exposure to DH results in an increase of both stored energy $(+70 \%)$ and peak strain $(+68 \%)$ at a roughly constant peak shear stress level.

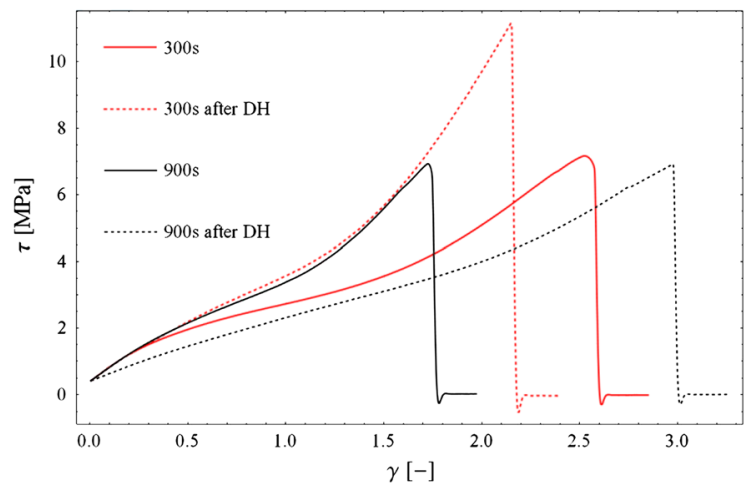

Figure 8. Compressive shear test results, showing the shear stress $\tau$ as a function of the shear strain $\gamma$ for ethylene-vinyl acetate samples for $300 \mathrm{~s}$ of curing or $900 \mathrm{~s}$ of curing (black curves), before damp-heat (plain curves) and after damp-heat (dashed curves). 
Table I. Adhesion indicators for ethylene-vinyl acetate at different curing times before and after degradation.

\begin{tabular}{lrlrl}
\hline & $\tau_{\max }[\mathrm{MPa}]$ & $\gamma_{\max }[-]$ & $E^{\mathrm{el}}\left[\mathrm{mJ} / \mathrm{mm}^{3}\right]$ & Failure \\
\hline EVA 300 s no DH & $7.3 \pm 0.3$ & $2.5 \pm 0.07$ & $9 \pm 0.17$ & Cohesive \\
EVA 300 s DH & $11.5 \pm 0.3$ & $2.1 \pm 0.1$ & $10.4 \pm 0.7$ & Adhesive \\
EVA 900 s no DH & $6.8 \pm 0.6$ & $1.7 \pm 0.1$ & $5.5 \pm 0.4$ & Adhesive \\
EVA 900 s DH & $7.1 \pm 0.4$ & $2.9 \pm 0.15$ & $9.4 \pm 0.6$ & Adhesive \\
\hline
\end{tabular}

EVA, ethylene-vinyl acetate; $\mathrm{DH}$, damp-heat.

\section{DISCUSSION}

\subsection{Effect of ethylene-vinyl acetate curing time on adhesion before and after degradation}

\subsubsection{Bulk deformation of the ethylene-vinyl acetate layer.}

The deformation of the studied EVA layers before failure at the interface can be described using a hyperelastic model accounting for medium and large deformations. Hyperelastic models for rubber elasticity state that the stress-strain relationship is driven by an entropy decrease attributable to the reduction of available conformations when the polymer chains are stretched [30]. This entropy reduction results in a restoring force opposing the deformation. Figure 10 shows a fit of the Lopez-Pamies hyperelastic model applied in pure shear [31] to EVA CST curves before degradation. The correlation is almost perfect with an error below $3 \%$ over the entire range of deformation.

The hyperelastic deformation of a crosslinked polymer can be divided into two regimes. At low deformations, the chains can elongate in between the crosslinking points, and the hyperelastic response is dominated by network conformation. In this regime, the curing level does not influence the hyperelastic behavior. At moderate and large deformations, the crosslinking points start to limit the chains extension, and the hyperelastic response becomes stiffer as more and more crosslinking points are stressed.

This two-regime behavior is clearly seen when comparing $300 \mathrm{~s}$ and $900 \mathrm{~s}$ EVA samples before degradation (Figure 10). The high gel content $(900 \mathrm{~s})$ leads to an overall increase of the hyperelastic curve slope as more crosslinking points are under solicitation.
Table II. Adhesion indicators for polyvinyl-butyral and ethylenevinyl acetate before and after degradation in damp-heat conditions.

\begin{tabular}{lrcrc}
\hline & $\tau_{\max }[\mathrm{MPa}]$ & $\gamma_{\max }[-]$ & $E^{\mathrm{e}}\left[\mathrm{mJ} / \mathrm{mm}^{3}\right]$ & Failure \\
\hline PVB before DH & $16.2 \pm 0.8$ & $2.2 \pm 0.1$ & $18.7 \pm 1.7$ & Adhesive \\
PVB after DH & $7.9 \pm 0.3$ & $1 \pm 0.02$ & $3.56 \pm 0.3$ & Adhesive \\
EVA before DH & $6.8 \pm 0.6$ & $1.7 \pm 0.1$ & $5.5 \pm 0.4$ & Adhesive \\
EVA after DH & $7.1 \pm 0.4$ & $2.9 \pm 0.15$ & $9.4 \pm 0.6$ & Adhesive
\end{tabular}

PVB, polyvinyl-butyral; DH, damp-heat; EVA, ethylene-vinyl acetate.

When the $300 \mathrm{~s}$ EVA samples are subjected to DH conditions, their hyperelastic behavior is modified and fits quite well with the response of $900 \mathrm{~s}$ EVA samples before degradation (Figure 8). This provides evidence of postcuring processing of the low gel content EVA samples. The remaining peroxide after the lamination process is activated during DH and allows the radical reaction (thus curing process) to be completed.

The behavior of $900 \mathrm{~s}$ EVA samples after DH is no longer similar to the other conditions in the low deformation regime. As a decrease in the crosslinking density because of $\mathrm{DH}$ exposure is very unlikely (owing to the great stability of the crosslinking points and the relatively low DH exposure time), this indicates that the $900 \mathrm{~s}$ samples experience a conformational change during degradation. Through the effect of temperature, the EVA network rearranges itself in a way such that subsequent chain stretching is facilitated. This may explain the softer response and the deviation in the low deformation regime as compared with the non-aged condition.

\subsubsection{Adhesion characterization.}

Using the hyperelastic model, we showed that the change in the CST curve shape for the degraded 300 s samples can be explained by a post-curing process (Section 5.1.1). The curing level of the degraded $300 \mathrm{~s}$ samples is then comparable with that of the $900 \mathrm{~s}$. The bulk behavior for both conditions is equivalent, and the difference between the calculated indicators is related to a change in interfacial adhesion.

Assuming the post-curing process of $300 \mathrm{~s}$ EVA samples is completed before the end of $70 \mathrm{~h} \mathrm{DH}$, an excess of radicals remains that can participate in a post-grafting reaction. Therefore, one expects an improvement of the

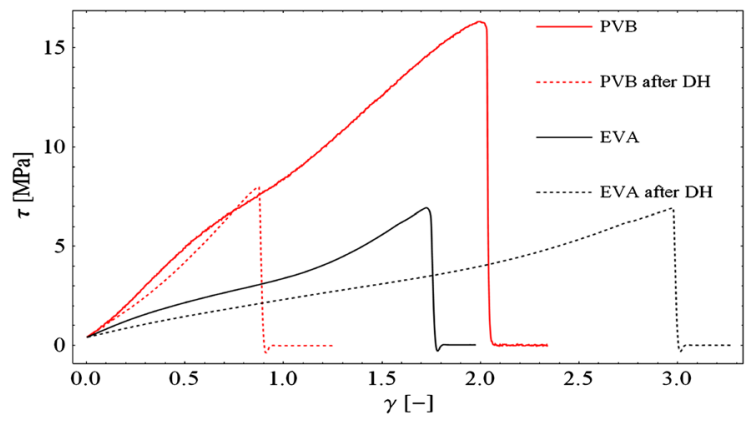

Figure 9. Representative compressive shear test results showing the shear stress as a function of the applied shear strain for polyvinyl-butyral and ethylene-vinyl acetate 900 s of curing (black curves), before (plain curves) and after degradation in damp-heat conditions (dashed curves). 


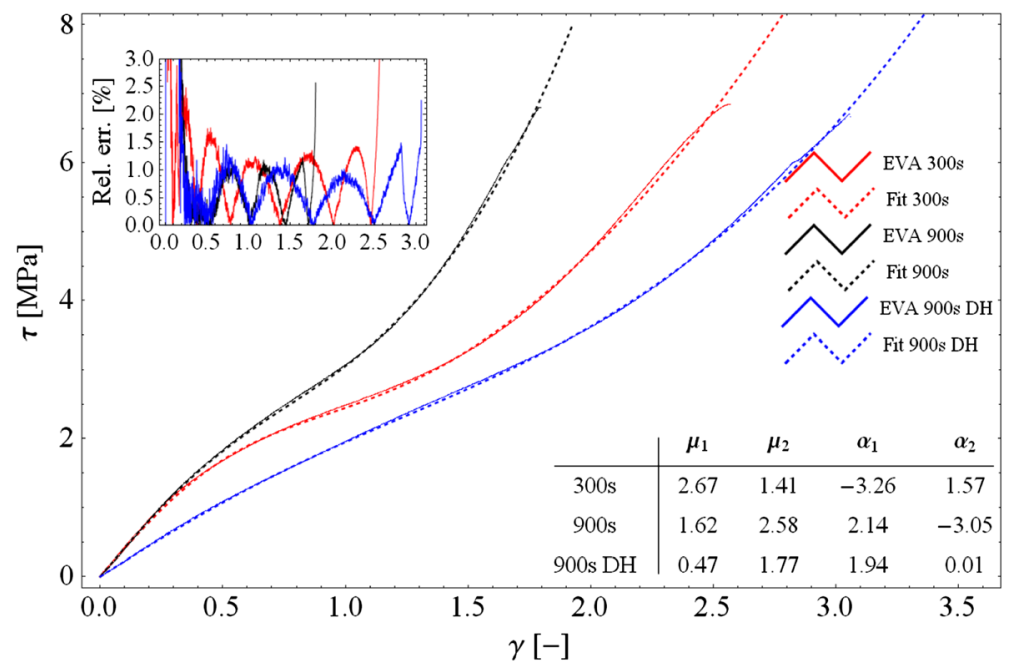

Figure 10. Hyperelastic fit (dashed curves) applied to compressive shear tests (plain curves) performed on ethylene-vinyl acetate with different curing times. Embedded table gives the values for the Lopez-Pamies model parameters.

interphase quality and thus an improvement of the interfacial adhesion as compared with the non-aged condition.

The post-grafting reaction uses radicals remaining after completion of the EVA curing process; it should also occur when subjecting the $900 \mathrm{~s}$ to $\mathrm{DH}$, as some non-activated peroxide remains in the polymer. Thus, an improvement of the adhesion for $900 \mathrm{~s}$ EVA samples should also be noticed after degradation.

Moreover, as the curing levels of $900 \mathrm{~s}$ before degradation and $300 \mathrm{~s}$ after degradation are equivalent, the amount of remaining peroxide should be the same in both conditions. Consequently, the post-grafting reaction should result in an equivalent interphase improvement, and the measured adhesion for both degraded samples should be similar.

Looking at the indicators given in Table III one observes that the peak stress for the degraded $300 \mathrm{~s}$ of curing $(11.5 \mathrm{MPa})$ is higher than that for the degraded $900 \mathrm{~s}$ (7.1 MPa), and that the peak strain follows the opposite trend, being 2.9 at $900 \mathrm{~s}$ and 2.1 at $300 \mathrm{~s}$. The stored energy is however of the same order of magnitude for both conditions, with a value around $10 \mathrm{~mJ} / \mathrm{mm}^{3}$.

Our results show that only the stored energy is able to describe the evolution of interfacial adhesion through degradation. Peak stress and peak strain do not only depend on the interface adhesion but also vary with the encapsulant bulk properties. For a given interfacial adhesion, the peak stress will increase as the polymer becomes stiffer (higher curing level, rigid conformation), whereas the shear strain follows the opposite trend. The adhesive failure of a CST sample occurs once a critical energy has been stored in the encapsulant layer and should therefore be described using an energy-controlled indicator such as the elastic deformation energy.

\subsection{Evolution of adhesion through damp- heat for polyvinyl-butyral and ethylene-vinyl acetate}

The strong drop in all adhesion indicators after DH degradation for PVB can be explained by the nature of the bonds. As presented in Section 3.2, adhesion between PVB and glass arises mostly from hydrogen bonds, which are sensitive to moisture. Water molecules can separate the hydrogen bonds between PVB and glass in order to adsorb in a liquid state. High temperature increases this effect as the diffusion coefficient of water vapor in PVB becomes larger.

As no plastic deformation has been observed in the PVB samples, the stored energy only contains a contribution from elastic deformation and can be used directly to describe the adhesion that drops from $18.7 \mathrm{~mJ} / \mathrm{mm}^{3}$ to $3.6 \mathrm{~mJ} / \mathrm{mm}^{3}$ through degradation.

As presented in Section 5.1.2, the situation is different for EVA cured for $900 \mathrm{~s}$. The stored energy initially at $5.5 \mathrm{~mJ} / \mathrm{mm}^{3}$ is much lower than that for PVB, showing that even though the established bonds in the case of EVA are stronger (covalent); their density is lower than that for

Table III. Summary of observations made for glass/ethylene-vinyl acetate/glass samples with different curing and damp-heat exposure times.

\begin{tabular}{lccclcrcc}
\hline & Failure & Curing & Conformation & Interphase grafting & Interface bonding & $\boldsymbol{\tau}_{\text {max }}[\mathrm{MPa}]$ & $\boldsymbol{\gamma}_{\max }[-]$ & $E^{\mathrm{el}}\left[\mathrm{mJ} / \mathrm{mm}^{3}\right]$ \\
\hline $300 \mathrm{~s}$ DH & Adhesive & Completed & Crosslinked network & Improved & Complete & 11.5 & 2.1 & 10.4 \\
$900 \mathrm{~s}$ & Adhesive & Completed & Crosslinked network & Partial & Complete & 6.8 & 1.7 & 5.5 \\
$900 \mathrm{~s}$ DH & Adhesive & Completed & Relaxed network & Improved & Complete & 7.1 & 2.9 & 9.4 \\
\hline
\end{tabular}

$\mathrm{DH}$, damp-heat. 
PVB. Exposure to DH conditions results in improved adhesion, with stored energy increasing to $9.4 \mathrm{~mJ} / \mathrm{mm}^{3}$. With this value being still lower than the initial stored energy for PVB, it indicates that even if the grafting process of silanes on EVA is complete, the density of established bonds is still lower than that for PVB.

\section{CONCLUSION}

A new CST design compatible with any universal tensile testing machine was developed. This testing method was used to characterize adhesion of standard encapsulants (PVB and EVA) to a glass substrate before and after degradation in $\mathrm{DH}$ conditions $\left(85^{\circ} \mathrm{C} / 85 \% \mathrm{RH}\right)$.

It is shown that during a CST, the layers of EVA exhibit a hyperelastic deformation until catastrophic delamination of the EVA/glass interface takes place. This indicates that the compressive shear test can be understood in terms of classical fracture mechanics theories, and more work is to be done to accurately describe the delamination initiation and propagation stages.

By testing EVA processed with different curing times before and after degradation, it is demonstrated that the encapsulant adhesion is neither accurately described by the peak stress at failure nor by the peak strain. Instead, we showed that delamination occurs once a critical energy is available to be released from the encapsulant to the interface. The strain energy stored in the encapsulant elastic deformation is thus proposed as a more reliable metric to measure adhesion. The developed CST analysis thus provides a quick and reliable tool to evaluate the adhesion energy of PV encapsulants to rigid substrates without the need of any additional test to be performed, and consequently allows to compare testing conditions where both the adhesive and the substrate properties are different.

We show that short exposure to $\mathrm{DH}(70 \mathrm{~h})$ has a positive effect on the adhesion of EVA to glass for both high and low curing times, whereas it has a detrimental effect on the adhesion of PVB to glass even if its initial adhesion is much higher than that of EVA. This effect can be explained by an improvement of the adhesion promoters grafting on the EVA chains during DH exposure. On the other hand, PVB forms mostly hydrogen bonds that are easily degraded by moisture leading to a large decrease in adhesion after DH.

Using the obtained results, guidelines can be drawn for the use of PVB and EVA in degradation conditions where the polymer becomes saturated. This can occur on a module edge during damp-heat exposure when no edge sealant is used. The subsequent loss of adhesion can increase the probability of failure in different IEC tests such as the breakage test (IEC 61730) or the hail test (IEC 61646). In this case, EVA is to be preferred to PVB because of the positive evolution of adhesion after degradation. However, PVB remains a wise choice with its initially higher adhesion, if moisture saturation can be prevented (e.g., using an edge sealing agent).

\section{ACKNOWLEDGEMENTS}

This study led in the framework of the ArchinSolar project, and the authors would like to thank the Center of Competence for Energy and Mobility (CCEM), the Swiss Federal Office for Energy (SFOE), Swiss Electric Research (SER) and the Services Industriels Genevois (SIG) for funding.

The authors would also like to thank the EPFL-ATMX workshop for samples preparation, Vincent Trachsel for his help with the samples testing, and Christian Schlumpf for careful manuscript reading.

\section{REFERENCES}

1. Czanderna AW, Pern FJ. Encapsulation of PV modules using ethylene vinyl acetate copolymer as a pottant: A critical review. Solar Energy Materials and Solar Cells 1996; 43: 101-108.

2. Oreski G, Wallner GM. Delamination behaviour of multi-layer films for PV encapsulation.. Solar Energy Materials and Solar Cells 2005; 89: 139-151.

3. Jorgensen GJ, Terwilliger KM, DelCueto JA, Glick SH, Kempe MD, Pankow JW, Pern FJ, McMahon TJ. Moisture transport, adhesion, and corrosion protection of PV module packaging materials. Solar Energy Materials and Solar Cells 2006; 90: 2739-2775.

4. Jorgensen G, Terwilliger K, Glick S, Pern J, McMahon T. Material testing for PV module encapsulation, NREL/ CP-520-33578, May 2003.

5. Van Dyk EE, Chamel JB, Gxasheka AR. Investigation of delamination in an edge-defined film-fed growth photovoltaic module. Solar Energy Materials and Solar Cells 2005; 88: 403-411.

6. Keller UW, Mortelmans H. Adhesion in laminated safety glass - What makes it work ?, Glass Processing Days, 13-16 June 1999.

7. Matthews FL, Kilty PF, Godwin EW. A review of the strength of joints in fibre-reinforced plastics. Composites January 1982; 29-37.

8. Herrera-Franco PJ, Drzal LT. Comparison of methods for the measurement of fibre/matrix adhesion in composites. Composites 1992; 23(1): 1-27.

9. Menningen M, Weiss H. Surface and Coatings Technology 1995; 76-77: 835-840.

10. Awajas F, Gilbert M, Kelly G, Fox B, Pigram PJ. Adhesion of polymers. Progress in Polymer Science 2009; 34: 948-968.

11. Sargent JP. Durability studies for aerospace applications using peel and wedge tests. International Journal of Adhesion and Adhesives 2005; 25: 247-256.

12. Moore DR. An introduction to the special issue on peel testing. International Journal of Adhesion and Adhesives 2008; 28: 153-157. 
13. ASTM Standard Test Method D6862-11 for 90 degree peel resistance of adhesives.

14. ASTM Standard Test Method D3983-98 for measuring strength and shear modulus of non-rigid adhesives by the thick adherend tensile-lap specimen.

15. Mendels D-A, Page SA, Leterrier Y, Månson J-AE, Nairn JA. European conference on composite materials, Brighton, UK, 4-7 June 2000.

16. Hadavina H, Kawashita L, Kinloch AJ, Moore DR, Williams JG. A numerical analysis of the elastic-plastic peel testEngineering Fracture Mechanics 2006; 73: 2324-2335.

17. Rahul-Kumar P, Jagota A, Bennison SJ, Saigal S. Interfacial failures in a compressive shear strength test of glass/polymer laminates. Acta Materialia 1999; 47(15): 4161-4169.

18. Schneider K, lauke B, Beckert W. Compression Shear Test (CST) - a convenient apparatus for the estimation of apparent shear strength of composite materials. Applied Composite Materials 2001; 8: 43-62.

19. Crocombe AD, Bigwood DA, Richardson G. Analysing joints for structural adhesive failure. International Journal of Adhesion and Adhesives 1990; 10(3): 167-178.

20. Papini M, Fernlund G, Spelt JK. The effect of geometry on the fracture of adhesive joints. International Journal of Adhesion and Adhesives 1994; 14(1): 5-13.

21. Pern FJ, Glick SH. Adhesion strength study of EVA encapsulants on glass substrates, NREL/CP-520-33558, May 2003.
22. Da Silva LFM, Rodrigues TNSS, Figueiredo MAV, de Moura MFSF, Chousal JAG. Effect of adhesive type and thickness on the lap shear strength. The Journal of Adhesion 2006; 82: 1091-1115.

23. Abaqus 6.8.1, Dassault Systèmes, 2004.

24. Kempe MD, Jorgensen GJ, Terwilliger KM, McMahon TJ, Kennedy CE, Borek TT. Acetic acid production and glass transition concerns with ethylene-vinyl acetate used in photovoltaic devices. Solar Energy Materials \& Solar Cells 2007; 91: 315-329.

25. Koenig JL, Boerio FJ, Plueddemann EP, Miller J, Willis PB, Cuddihy EF. Chemical bonding technology: direct investigation of interfacial bonds, JPL Publication 86-6, January 1986.

26. Li H-Y et al. submitted 2011.

27. Klemchuk P, Ezrin M, Lavigne G, Halley W, Agro JS. Investigation of the degradation and stabilization of EVAbased encapsulant in field-aged solar energy modules. Polymer Degradation and Stability 1997; 55: 347-365.

28. Vogt M. Etimex review, 28th March 2006.

29. Li H-Y, Lange RFM, Luo Y, Polo R, Zahnd J. The lamination of (multi)crystalline and thin film based photovoltaic modules. Progress in Photovoltaics: Research and Applications. 2011. DOI: 10.1002/pip.1175

30. Kausch H-H. Traité des Matériaux 14. PPUR: Lausanne, 2001.

31. Lopez-Pamies O. A new I1-based hyperelastic model for rubber elastic materials. Comptes Rendus Mécanique 2010; 338: 3-11. 